\title{
High-energy and very high-energy gamma-ray emission from the magnetar SGR $1900+14$ outskirts
}

\author{
Bohdan Hnatyk, ${ }^{a}$ Roman Hnatyk, ${ }^{a}$ Valery Zhdanov ${ }^{a}$ and Vadym Voitsekhovskyi ${ }^{a, *}$ \\ ${ }^{a}$ Taras Shevchenko National University of Kyiv, Astronomical Observatory \\ Observatorna st. 3, Kyiv, Ukraine \\ E-mail: bohdan_hnatyk@ukr.net, roman_hnatyk@ukr.net, \\ valeryzhdanov@gmail.com, v.v.voitsekhovskyi@gmail.com
}

One of the unsolved problems in cosmic ray (CR) physics is the determination of sources and acceleration mechanism(s) of Galactic $\left(E \lesssim 10^{18}\right) \mathrm{eV}$ and extragalactic $\left(E \gtrsim 10^{17} \mathrm{eV}\right) \mathrm{CR}$. Ultra high energy CR (UHECR, $E>10^{18} \mathrm{eV}$ ) are believed to be of extragalactic origin, but some contribution from transient Galactic sources is possible. So, magnetar wind nebulae (MWNe), created by newborn millisecond magnetars, and magnetar giant flares are PeVatron candidates and even potential sources of UHECR. Promising signature of effective acceleration processes in magnetars' neighbourhoods should be nonthermal high-energy and very high-energy $\gamma$-ray emission due to pp collisions with a subsequent pion decay (hadronic scenario) and inverse Compton scattering of low energy background photons by ultrarelativistic electrons and positrons (leptonic scenario).

In this work we explain the HE and VHE $\gamma$-ray emission from the vicinity of the magnetar SGR 1900+14 - potential Galactic source of $E>10^{20} \mathrm{eV}$ triplet - by the hadronic and leptonic emission of cosmic rays accelerated in a magnetar-related Supernova remnant (SNR) and/or MWN. To this end we have carried out a simulation of the observed HE and VHE $\gamma$-ray emission, spatially coincident with the magnetar SGR 1900+14. The extended Fermi-LAT source 4FGL J1908.6+0915e, the extended H.E.S.S. source candidate HOTS J1907+091 and the point-like HAWC TeV source $3 \mathrm{HWC}$ J1907+085 were considered. We show that the observed $\gamma$-ray emission from the magnetar SGR 1900+14 outskirts can be explained by a (still undetected) magnetarconnected super-luminous Supernova (Hypernova) remnant and/or a MWN created by newborn millisecond magnetar with a large initial rotational energy $E_{\mathrm{rot}} \sim 10^{52} \mathrm{erg}$.

$37^{\text {th }}$ International Cosmic Ray Conference (ICRC 2021)

July 12th-23rd, 2021

Online - Berlin, Germany

\footnotetext{
*Presenter
} 


\section{Introduction}

Magnetars - young neutron stars with extremely high magnetic fields $\sim 10^{14}-10^{15} \mathrm{G}$ - exhibit persistent X-ray $-\gamma$-ray activity: persistent/transient quiescent super-luminous X-ray emission, together with short (milliseconds-seconds) bursts and longer (weeks-months) outbursts (anomalous $\mathrm{X}$-ray pulsars, AXPs), repeated hard X-ray - soft $\gamma$-ray flares (soft gamma-repeaters, SGRs), including rare giant flares (GFs) - the short $(\sim 0.1 \mathrm{~s})$ hard X-ray - soft $\gamma$-ray bursts with luminosities $\sim 10^{44}-10^{47} \mathrm{erg} \mathrm{s}^{-1}$ and released energies $\sim 10^{44}-10^{46} \mathrm{erg}$. Approximately one GF with $E>10^{44}\left(10^{46}\right)$ erg is expected to occur every 6 (200) years [4]. Until now, three GFs have been detected in our Galaxy and LMC (including from magnetar SGR1900+14) and three GFs from the outside the Local Group [18].

Only a few percent of newborn pulsars are magnetars, and only a very small fraction, $10^{-3}-10^{-2}$ of magnetars is expected to have millisecond initial periods $P_{\mathrm{i}} \sim 10^{-3}$ s. Huge initial rotational kinetic energy of a newborn millisecond magnetar $E_{\mathrm{rot}} \approx 2 \times 10^{52} P_{\mathrm{i},-3}^{-2}$ erg can reveal itself as a magnetar wind nebula (MWN) and/or a Hypernova via additional energization of a Supernova ejecta by magnetic dipole radiation with spin-down luminosity $L_{\mathrm{sd}, \mathrm{i}} \approx 10^{49} B_{15}^{2} P_{\mathrm{i},-3}^{-4} \mathrm{erg} \mathrm{s}^{-1}$ and an initial spin-down timescale $t_{\mathrm{sd}, \mathrm{i}}=E_{\mathrm{rot}} / L_{\mathrm{sd}, \mathrm{i}} \approx 2 \times 10^{3} B_{15}^{-2} P_{\mathrm{i},-3}^{2} \mathrm{~s}[3,6,7,17]$.

Both magnetar GFs and MWNe of newborn millisecond magnetars are potential sources of UHECR [5, 8]. For example, magnetar SGR1900+14 with detected GF and position in the sky close to $E>10^{20} \mathrm{eV} \mathrm{CR}$ triplet (Fig. 1a) is a promising candidate on MWN/Hypernova-related magnetar of millisecond initial period with corresponding observational signatures in nonthermal emission from their outskirts [10-13].

In this work we analyse HE (Fermi-LAT) and VHE (H.E.S.S., HAWC) gamma-ray observational data of the magnetar SGR 1900+14 environment (Fig. 1b) and explain them in the model of a Hypernova-like explosion of a progenitor massive star with contribution of $10^{52}$ erg rotational energy of newborn fast rotating magnetar SGR 1900+14 to the Hypernova energy reserve and to the MWN formation.

\section{SGR 1900+14 and its neighbourhood: observations}

Untill now magnetar SGR 1900+14 in massive star cluster Cl1900+14 $\left(l=43.02^{\circ}, b=0.77^{\circ}\right.$, period $\mathrm{P}=5.2 \mathrm{~s}$, characteristic age $\tau_{c}=0.9 \mathrm{kyr}$, distance $d=12.5 \mathrm{kpc}$, giant flare in 1998 [15]) is detected only in X-ray- soft gamma -ray band and no signatures of an adjacent SNR or MWN were detected yet in the radio - X-ray bands.

In the HE $\gamma$-ray band SGR 1900+14 is overlapped by extended source 4FGL J1908.6+0915e of the Fermi-LAT Fourth Source Catalogue $\left(l=43^{\circ} .12, b=0^{\circ} .43\right.$, radius of $\left.\Theta=0^{\circ} .6\right)$ with overall significance only $4.58 \sigma$ [1] (Fig. 1b). In the VHE $\gamma$-ray band SGR 1900+14 is overlapping with the H.E.S.S. extended source candidate (hotspot) HOTS J1907+091 $\left(l=42^{\circ} .88, b=0^{\circ} .69\right.$, radius of $\Theta=0^{\circ} .17$ ) with cross-check TS $=43$ [9]. In the 3HWC HAWC Observatory Gamma-Ray Catalogue [2] the nearest to SGR $1900+14$ (a separation of $\approx 0^{\circ} .75$ ) is the $\mathrm{TeV}$ point-like source 3 HWC J1907+085 $\left(l=42^{\circ} .35, b=0^{\circ} .44\right)$ with a somewhat low $(\mathrm{TS}=75.5)$ peak on an extended background spot (Fig. 1b). 


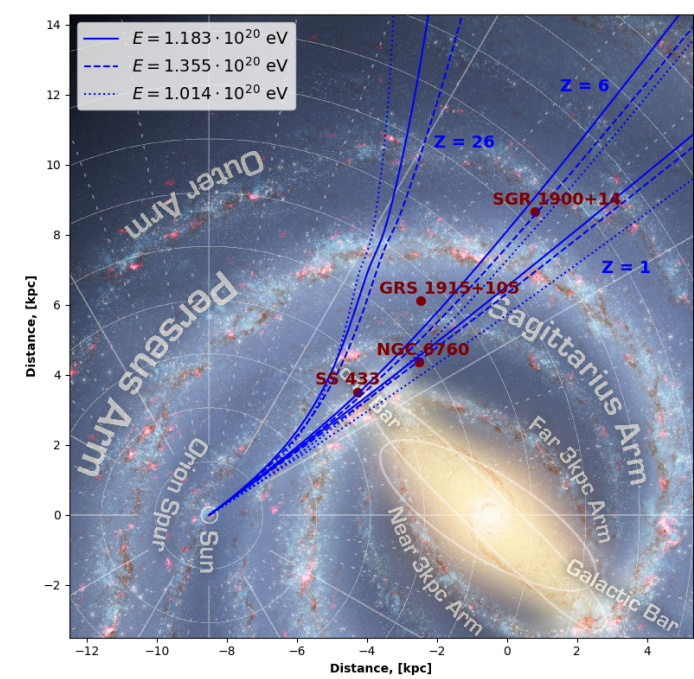

(a)

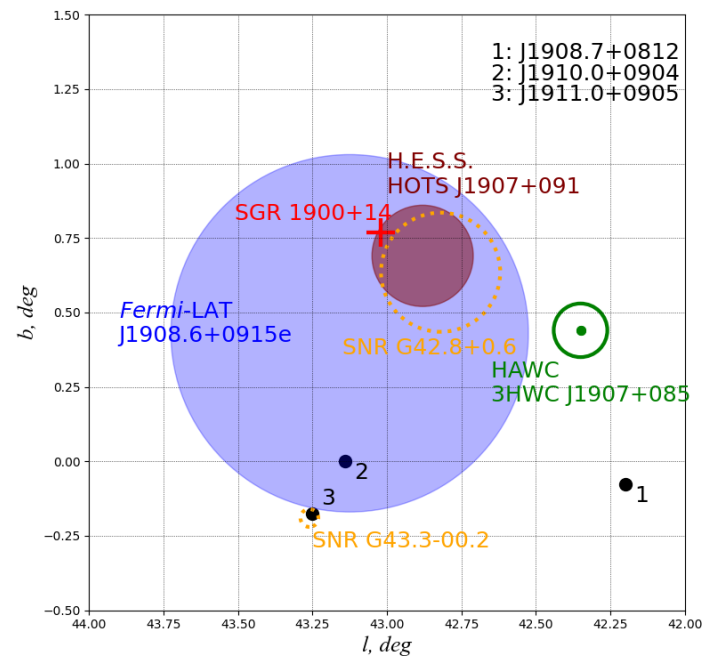

(b)

Figure 1: Magnetar SGR 1900+14 neighbourhood. Left (a): backwards trajectories of $E>10^{20} \mathrm{eV}$ triplet in the magnetar region. The background image: courtesy NASA/JPL-Caltech/R. Hurt, SSC/Caltech. Right (b): HE and VHE $\gamma$-ray sources in the magnetar region. The Fermi-LAT extended source 4FGL J1908.6+0915e (the light blue circle), the three extragalactic point-like 4FGL sources (the black bullets), the H.E.S.S. extended source HOTS J1907+091 (the brown circle) and the HAWC point-like source 3HWC $\mathrm{J} 1907+085$ (the green bullet with the $1 \sigma$ error box) are presented. Two SNRs in the field (the orange rings) are not physically connected with the region considered.

All these HE and VHE $\gamma$-ray sources can be signatures of magnetar- connected SNR/MWN. Moreover, association of SGR1900+14 (with an improved age $\tau_{c} \approx 2.4 \mathrm{kyr}$ estimate from new data for $P=5.22669(3) \mathrm{s}$ and $\dot{P}=3.3 \times 10^{-11} \mathrm{~s} \mathrm{~s}^{-1}$ [19]) with the 4 BCE "po star" [20] suggests a Hypernova type of a magnetar-related Supernova with favourable conditions for UHECR acceleration [14]. Necessary for Hypernova $\sim 10^{52}$ erg of ejecta's energy can be provided by newborn millisecond magnetar SGR1900+14 with $E_{\text {rot }} \approx 10^{52}$ erg in the form of spherically symmetric or jet-like collimated magnetar wind that additionally energizates Supernova ejecta and following Hypernova remnant (HNR) up to $E_{k i n} \sim 10^{52} \mathrm{erg}$.

Moreover, another scenario is possible when considerable part of newborn MWN plasma destroys and protrudes the ejecta shell and evolves till present to a large size (tens of parsecs) MWN ahead the fragmented debrises of initial ejecta. Depending on ISM density a new shell from swept up ISM plasma may be formed.

In both cases the high pressure of MWN plasma inside SN ejecta results in R-T instability and fragmentation of ejecta at distances 2-4 pc from SN outburst. Morphology of infrared (IR) dust emission of massive star cluster - SGR1900+14 cradle - supports the Hypernova model and $\sim 2 \mathrm{kyr}$ age of SNR (Fig. 2).

SGR 1900+14-related HNR/MWN should manifest themselves as PeVatrons with nonthermal emission from the radio to $\mathrm{TeV} \gamma$ - ray band [12]. 


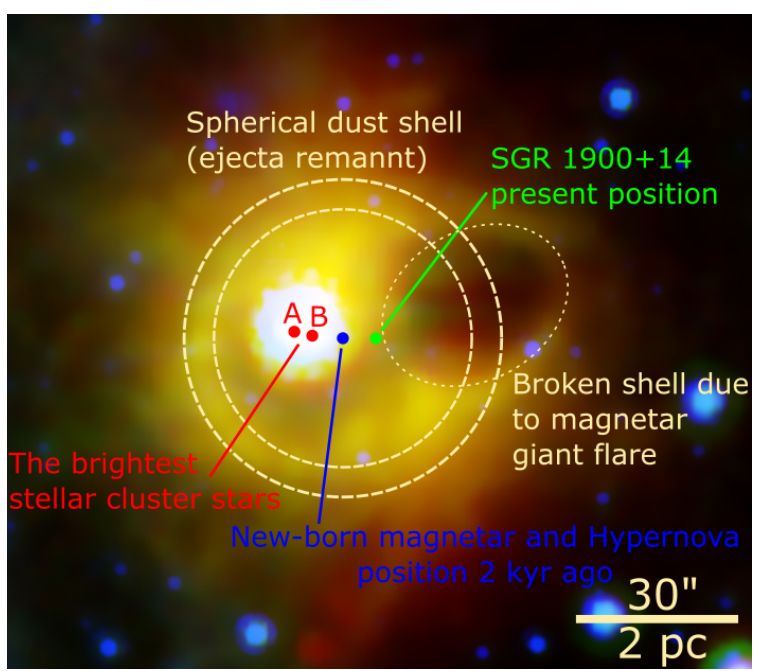

Figure 2: The background image is a Spitzer IR map of SGR 1900+14 environment with a colour coding: blue $(8 \mu \mathrm{m})$, green $(16 \mu \mathrm{m})$, and red $(24 \mu \mathrm{m})$ (courtesy NASA/JPL/Caltech/S. Wachter (Caltech-SSC)). In our model the observed IR dust emission of massive stellar cluster dominates by a spherical shell of dusty ejecta debris and the broken shell is created by the anisotropic giant flare of the magnetar.

\section{Gamma-ray emission of the SGR 1900+14 neighbourhood in the Hypernova remnant case}

In our Hypernova model the HNR with $E_{\mathrm{HNR}} \sim 10^{52}$ erg evolves inside the low-density wind bubble $\left(n_{\mathrm{w}} \sim 10^{-2} \mathrm{~cm}^{-3}, B_{\mathrm{w}} \lesssim 3 \mu \mathrm{G}\right)$. At present $\left(t_{\mathrm{HNR}} \approx 2 \mathrm{kyr}\right)$ HNR is at the ST stage (the shell radius $R_{\mathrm{HNR}} \approx 24 \mathrm{pc}$, the shock velocity $V_{\mathrm{HNR}}=0.4 R_{\mathrm{HNR}} / t_{\mathrm{HNR}} \approx 5000 \mathrm{~km} \mathrm{~s}^{-1}$ ) and shock-accelerated CR protons $(\mathrm{i}=\mathrm{p})$ and electrons $(\mathrm{i}=\mathrm{e})$ obey exponential cut-off power-law spectra

$$
N_{\mathrm{i}}\left(E_{\mathrm{i}}\right)=N_{0, \mathrm{i}}\left(E / E_{0, \mathrm{i}}\right)^{-\gamma_{\mathrm{i}}} \exp \left(-E / E_{\text {cut }, \mathrm{i}}\right)
$$

with $N_{\mathrm{e}}(E)=K_{\mathrm{ep}} N_{\mathrm{p}}(E)$, where electron-to-proton ratio $K_{\text {ep }} \lesssim\left(m_{\mathrm{e}} / m_{\mathrm{p}}\right)^{1 / 2} \approx 0.02$ [16].

Observed $\gamma$-ray emission is generated mainly by the hadronic mechanism (pp-collisions with the subsequent neutral pion decay) in the dense $\left(n_{\mathrm{sh}} \sim 10 \mathrm{~cm}^{-3}\right)$ extended $(R \sim 30-40 \mathrm{pc})$ shell-like halo of the swept up stellar cluster gas of $\sim 3 \times 10^{4} \mathrm{M}_{\odot}$.

In order to estimate the necessary Hypernova remnant and cosmic ray parameters to explain the Fermi-LAT and H.E.S.S. sources (the HAWC source flux is similar to that of the H.E.S.S. source) we have carried out the NAIMA-fitting [21] of Fermi-LAT and H.E.S.S. spectral energy distributions (SEDs) in the HNR model (Fig. 3, Table 1). Herewith we present the H.E.S.S. 1-10 $\mathrm{TeV}$ integral flux $F(E>1 \mathrm{TeV})=4.3 \times 10^{-13} \mathrm{~cm}^{-2} \mathrm{~s}^{-1}$ as a differential power-law spectrum with a photon index $\gamma=2.3 \pm 0.2$ and $\delta F\left(E_{0}\right)=0.2 F\left(E_{0}\right)$ at $E_{0}=3 \mathrm{TeV}$. The Hypernova model explains observed SED with the Hypernova-type $\left(W_{S N R} \approx 10^{52} \mathrm{erg}\right.$ ) total energy of the proton component $W_{p} \approx 0.05 W_{S N R} \approx 5 \times 10^{50} \mathrm{erg}$ and an enhanced number density $n_{H} \approx 10 \mathrm{~cm}^{-3}$ of ISM. 


\section{Gamma-ray emission of the SGR 1900+14 neighbourhood in the MWN case}

Powerful relativistic magnetar wind can destroy and penetrate the Hypernova ejecta and afterwards support an expansion of MWN with $E_{\mathrm{MWN}} \lesssim E_{\mathrm{rot}} \approx 10^{52} \mathrm{erg}$ ahead ejecta's debris inside the low-density wind bubble till present. Electrons and positrons are accelerated at a termination shock (TS) or in magnetic reconnection processes and the present relativistic leptons in MWN are the result of the time-dependent acceleration and cooling/escaping/advection processes. Typical exponential cut-off broken power-law spectrum is expected in this case

$$
N_{\mathrm{e}}(E)=\left\{\begin{array}{r}
N_{0, \mathrm{e}}\left(E / E_{0, \mathrm{e}}\right)^{-\gamma_{1, \mathrm{e}}}, E<E_{\mathrm{br}, \mathrm{e}} \\
N_{0, \mathrm{e}}\left(E_{\mathrm{br}, \mathrm{e}} / E_{0, \mathrm{e}}\right)^{\left(\gamma_{2, \mathrm{e}}-\gamma_{1, \mathrm{e}}\right)}\left(E / E_{0, \mathrm{e}}\right)^{-\gamma_{2, \mathrm{e}}} \\
\quad \times \exp \left(-E / E_{\mathrm{cut}, \mathrm{e}}\right), E \geq E_{\mathrm{br}, \mathrm{e}}
\end{array}\right.
$$

with $\gamma_{1, \mathrm{e}}<2$ for $E \leq E_{\mathrm{br}, \mathrm{e}}$ and $\gamma_{2, \mathrm{e}}>2$ for $E \geq E_{\mathrm{br}, \mathrm{e}}$,

In MWN case electrons and positrons generate observed $\gamma$-ray emission by the inverse Compton (IC) scattering of the background CMB, IR and star light (SL) photons (leptonic mechanism) (Fig. 4).

In order to estimate the necessary MWN and leptonic cosmic ray parameters to explain the Fermi-LAT and H.E.S.S. sources we have carried out NAIMA-fitting of Fermi-LAT and H.E.S.S. SEDs in the MWN model (Fig 4, Table 1). MWN model explains observed SEDs in different variants of leptonic cosmic ray spectrum (exponential cut-off broken power-law (ECBPL), twoelectron ECPL populations etc.) only with unusually large total energy of CR leptons $W_{e} \approx$ $0.05 W_{\text {rot }} \approx 5 \times 10^{50} \mathrm{erg}$. Taking into account radiative and adiabatic energy losses, large value of $W_{e}$ confirms the large amount $\left(\sim 10^{52} \mathrm{erg}\right)$ of energy transfered to MWN from newborn millisecond magnetar.

To summarise, in both cases of HNR/MWN the observational data can be explained only when the total energy of cosmic rays ( protons or electrons) is $W_{C R} \approx 5 \times 10^{50} \mathrm{erg}$, that is, corresponds to the millisecond period of a newborn magnetar (Table 1). Moreover, in HNR and MWN cases the radio- and X-ray non-detection corresponds to a low value of magnetic field ( $B \lesssim 2 \mu \mathrm{G}$ ) in the magnetar neighbourhood.

Table 1: HNR and MWN NAIMA-fitted models of SED from SGR 1900+14 neighbourhood

\begin{tabular}{|c|c|c|c|c|c|}
\hline \multirow[t]{2}{*}{ Parameter } & \multicolumn{2}{|c|}{ HNR: PL and ECPL spectra $(i=p)$} & \multirow{2}{*}{$\frac{\mathrm{MWN}: \mathrm{ECBPL}(\mathrm{i}=\mathrm{e})}{\mathrm{ECBPL}}$} & \multicolumn{2}{|c|}{ MWN:Two electron population spectra $(\mathrm{i}=\mathrm{e})$} \\
\hline & PL & ECPL & & ECPL \#1 & ECPL \#2 \\
\hline$E_{\min }[\mathrm{GeV}]($ fixed $)$ & 1 & 1 & 1 & 1 & 1 \\
\hline$E_{\max }[\mathrm{GeV}]($ fixed) & $1 \mathrm{e} 6$ & $1 \mathrm{e} 6$ & $1 \mathrm{e} 6$ & $1 \mathrm{e} 6$ & $1 \mathrm{e} 6$ \\
\hline$N_{0, \mathrm{i}}[1 / \mathrm{eV}]$ & $(1.18 \pm 0.04) \mathrm{e} 35$ & $(1.42 \pm 0.05) \mathrm{e} 37$ & $(1.90 \pm 0.12) \mathrm{e} 40$ & $(4.41 \pm 0.51) \mathrm{e} 38$ & $(8.81 \pm 0.44) \mathrm{e} 34$ \\
\hline$E_{0, \mathrm{i}}[\mathrm{TeV}]$ & $3.93 \pm 0.1$ & $0.78 \pm 0.01$ & $0.19 \pm 0.01$ & $1.91 \pm 0.19$ & $1.81 \pm 0.19$ \\
\hline$E_{\mathrm{br}, \mathrm{i}}[\mathrm{TeV}]$ & - & - & $0.0047 \pm 0.0003$ & - & - \\
\hline$E_{\text {cut,i }}[\mathrm{TeV}]$ & - & $185.2 \pm 9.5$ & $396.7 \pm 41.7$ & $0.0096 \pm 0.0008$ & $9.99 \pm 1.06$ \\
\hline$\gamma_{1, \mathrm{i}}$ & $2.55 \pm 0.01$ & $2.41 \pm 0.03$ & $1.49 \pm 0.07$ & $1.65 \pm 0.11$ & - \\
\hline$\gamma_{2, \mathrm{i}}$ & - & - & $3.04 \pm 0.06$ & - & $2.58 \pm 0.17$ \\
\hline$W_{\mathrm{p}}$ [erg] & $5.03 \mathrm{e} 50$ & $5.12 \mathrm{e} 50$ & - & - & - \\
\hline$W_{\mathrm{e}}[\mathrm{erg}]$ & $1.04 \mathrm{e} 49$ & $2.16 \mathrm{e} 48$ & $3.60 \mathrm{e} 50$ & $5.20 \mathrm{e} 50$ & $6.08 \mathrm{e} 49$ \\
\hline$n_{\mathrm{H}}\left[\mathrm{cm}^{-3}\right]$ & $11.42 \pm 0.66$ & $9.81 \pm 0.35$ & - & - & - \\
\hline$K_{\text {ep }}$ & 0.02 (fixed) & $0.0041 \pm 0.0002$ & - & - & - \\
\hline
\end{tabular}




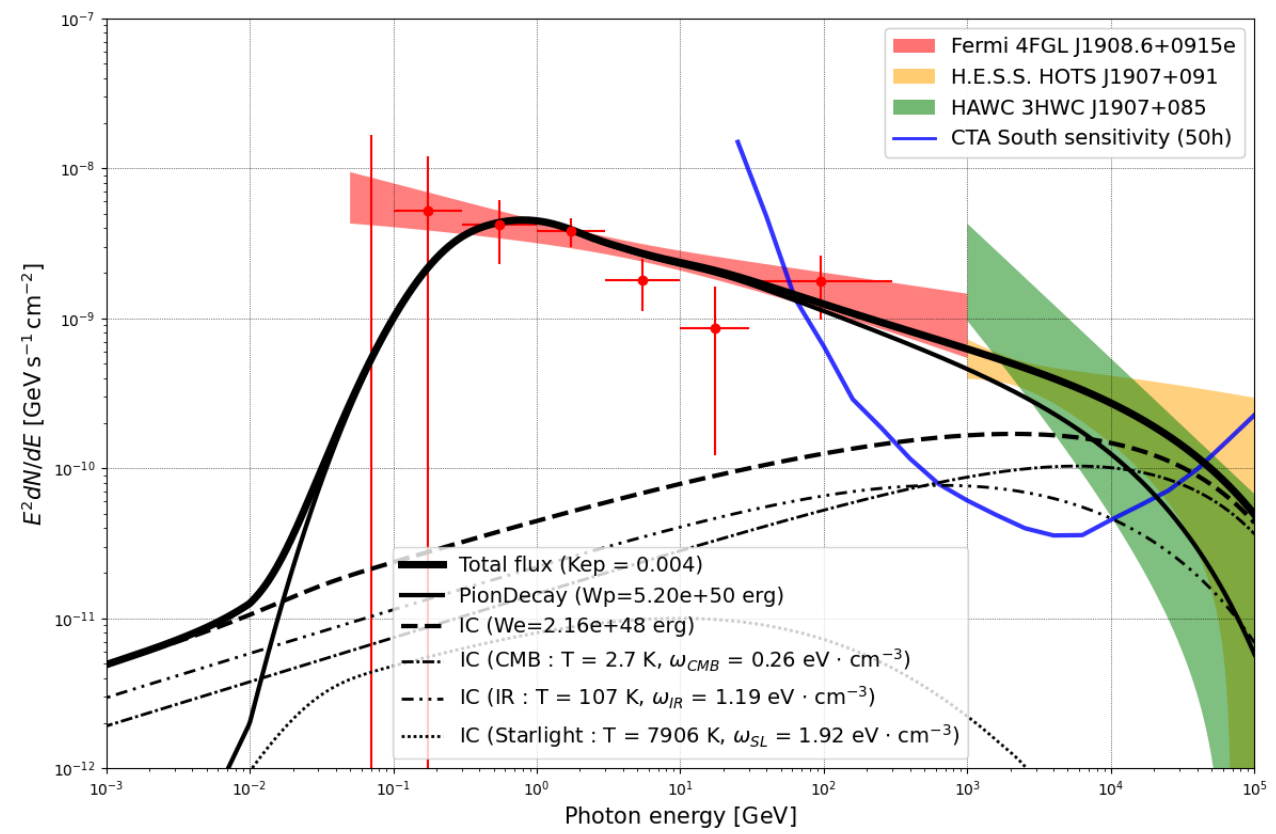

Figure 3: Modelled SED of the magnetar SGR 1900+14 neighbourhood in the HNR model. NAIMA-fitted parameters are given in Table 1 (the case of HNR shock accelerated proton and electron exponential cut-off power-law spectra with free $K_{\text {ep }}$ ). Observations of Fermi-LAT 4FGL J1908.6+0915e (in red), H.E.S.S. HOTS J1907+091 (in blue) and HAWC 3HWC J1907+085 (in green) are presented. The total gamma-ray flux is a sum of hadronic (pp collisions with the subsequent neutral pion decay) and leptonic (IC scattering of electrons on CMB, IR and SL background photons) contributions. The blue line represents the sensitivity of the CTA South array for a zenith angle $z=40^{\circ}$

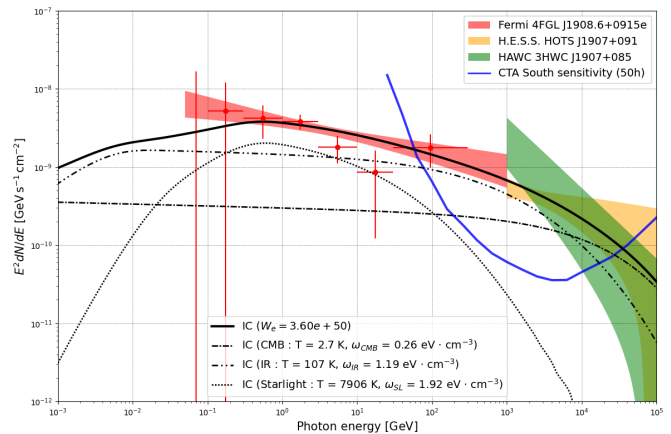

(a)

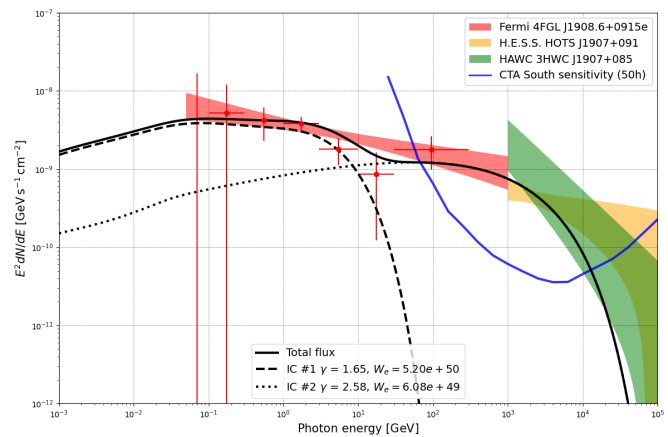

(b)

Figure 4: Modelled SED of the magnetar SGR 1900+14 neighbourhood in the MWN model. (a) The case of MWN termination shock/reconnection accelerated leptons with an exponential cut-off broken power-law spectrum. The total gamma-ray flux is a sum of the contributions from IC scattering of leptons on CMB, IR and SL background photons. The rest of the data is the same as in Fig. 3. (b) Same as in (a) but for two electron populations with exponential cut-off power-law spectra. For both populations Only the total gamma-ray fluxes as sums of the contributions from IC scattering of leptons on CMB, IR and SL background photons are presented. NAIMA-fitted parameters are given in Table 1. 


\section{Summary}

We have explained the observed $\gamma$-ray emission from the magnetar SGR 1900+14 neighbourhood in a model of magnetar-connected HNR and MWN created by an energy supply to a SN ejecta from a fast-rotating newborn magnetar with initial rotational energy $E_{\text {rot }} \sim 10^{52} \mathrm{erg}$. The new X-ray data of SGR 1900+14 observations support age estimate $\sim 2 \mathrm{kyr}$ and agree with Hypernova type of a visually detected (ancient Chinese records on 4 BC Apr 24) distant (12.5 kpc) Supernova outburst. Additional confirmation of Hypernova type of SN follows from an absence of SNR/MWN signatures in the recent radio- to X-ray observations due to high initial velocity of Hypernova shell and low density of wind bubble interior, in which shell is still expanding.

In the HNR model, most of the magnetar rotational energy is transformed to the Hypernova shell, now at an adiabatic stage of evolution. Shock accelerated nuclei and electrons are diffusively spread over the HNR shock and the $\gamma$-ray emission is generated mainly by the pp collisions with subsequent neutral pion decay in the dense $\left(n_{s h} \sim 10 \mathrm{~cm}^{-3}\right)$ extended shell-like halo of swept up gas $\left(\sim 3 \times 10^{4} \mathrm{M}_{\odot}\right)$ of massive stellar cluster-SGR $1900+14$ cradle. In the TeV $\gamma$-ray band the contribution of the IC scattering of background CMB, IR and SL photons by shock accelerated electrons can be important.

If the powerful relativistic magnetar wind effectively penetrates the Hypernova ejecta, the resulting energy-dominated MWN with energy loss corrected reserve $E_{M W N} \lesssim E_{\text {rot }} \sim 10^{52} \mathrm{erg}$ expands inside the low-density wind bubble till present. Electrons and positrons, accelerated at the TS or in magnetic reconnection processes, generate $\gamma$-ray emission by the IC scattering of background CMB, IR and SL photons.

Observational fluxes of the HE and VHE $\gamma$-ray emission from the SGR 1900+14 region can be explained in both- HNR and MWN models (Table 1, Figs. 3, 4). The main challenge of these models is the requirement of the considerably higher than the ordinary SNR/PWN value of total cosmic ray energy $W_{\text {cr,p }} \approx W_{\text {cr,e }} \sim 5 \times 10^{50} \mathrm{erg}$.

\section{Acknowledgements}

The Milky Way Galaxy image: courtesy NASA/JPL-Caltech/R. Hurt, SSC/Caltech. The Spitzer IR image of the SGR 1900+14 environment: courtesy NASA/JPL/Caltech/S. Wachter (Caltech-SSC).

\section{References}

[1] S. Abdollahi et al., ApJS 247 (2020) 33

[2] A. Albert et al. arXiv:2007.08582

[3] C. R. Angus et al., MNRAS 487 (2019) 2215

[4] P. Beniamini et al., MNRAS 487 (2019) 1426

[5] P. Blasi et al., ApJ 533 (2000) L123 
[6] N. Bucciantini et al., MNRAS, 380 (2007) 1541

[7] R. C. Duncan, Thompson C., ApJ 392 (1992) L9

[8] K. Fang et al., ApJ 878 (2019) 34

[9] H. E. S. S. Collaboration, A\&A 612 (2018) A1

[10] R. Gnatyk, Kinemat. Phys. Celest. Bodies 32 (2016) 1

[11] R. Gnatyk, Kinemat. Phys. Celest. Bodies 34 (2018) 167

[12] B. Hnatyk, R. Hnatyk, V. Zhdanov, V. Voitsekhovskyi, arXiv:2009.06081

[13] R. Hnatyk, V. Voitsekhovskyi, Kinemat. Phys. Celest. Bodies 36 (2020) 129

[14] K. Kotera, A. V. Olinto, ARA\&A 49 (2011) 119

[15] S. A. Olausen, V. M. Kaspi, ApJS 212 (2014) 6

[16] M. Pohl, A\&A 270 (1993) 91

[17] T. Sukhbold, Thompson T. A., MNRAS 472 (2017) 224

[18] D. Svinkin et al., Nature 589 (2021) 211

[19] T. Tamba et al., PASJ 71 (2019) 90

[20] Z. Wang et al., ApJ 569 (2002) L43

[21] V. Zabalza, arXiv:1509.03319 